\title{
Adaptación psicométrica de la Batería de Evaluación de los Procesos Lectores Revisada (PROLEC-R)
}

\section{Psychometric adaptation of the Assessment Battery of Reading Processes Reviewed (PROLEC-R)}

\author{
Nidia Cayhualla $^{1 \text { ab }}$, Daniela Chilón ${ }^{2 a c}$ \& Rolando H. Espíritu ${ }^{3 a d^{*}}$ \\ ${ }^{1}$ Centro Peruano de Audición, Lenguaje y Aprendizaje, Lima, Perú.
}

\begin{abstract}
${ }^{a}$ Magíster en Educación con Mención en Dificultades de Aprendizaje por la Pontificia Universidad Católica del Perú. ${ }^{b}$ Licenciada en la Especialidad de Educación Primaria y Ciencias Religiosas por la Universidad Marcelino Champagnat. ${ }^{\mathrm{C}}$ Licenciada en la Especialidad de Educación Primaria por la Universidad Nacional Mayor de San Marcos. ${ }^{\mathrm{d}}$ Psicólogo por la Universidad Nacional Mayor de San Marcos, se desempeña como Coordinador Académico y docente de la Carrera de Psicología de la Universidad San Ignacio de Loyola.
\end{abstract}

Recibido: 06-02-13

Aprobado: $25-08-13$

*Correspondencia

Email: respiritu@usil.edu.pe

\section{Citar Como:}

Cayhualla, N., Chilón, D., \& Espíritu, R. (2013). Adaptación psicométrica de la Batería de Evaluación de los Procesos Lectores Revisada (PROLEC-R). Propósitos y Representaciones, 1(1), 39-57. doi: http://dx.doi.org/10.20511/ pyr2013.v $\ln 1.3$ 


\section{Resumen}

El presente estudio tiene como objetivo la adaptación psicométrica de la Batería de Evaluación de los Procesos Lectores Revisada (PROLEC-R). Se aplicó la batería adaptada lingüística y pictográficamente a una muestra compuesta por 504 estudiantes, varones y mujeres, pertenecientes a 14 instituciones educativas de Lima metropolitana, siete particulares y siete estatales. Se obtuvo como resultados que las puntuaciones del PROLEC-R adaptado son confiables, calculadas a través del método de consistencia interna (alfa de Cronbach) por subtest y total. Así mismo evidencia validez de contenido (juicio de expertos), criterio (validez concurrente) y constructo (análisis factorial confirmatorio).

Palabras clave: Lectura, procesos lectores, confiabilidad, validez

\section{Summary}

The present study aims at adapting psychometric Assessment Battery Revised Readers Processes (PROLEC-R). The Battery applied linguistics and pictographically adapted to a group of 504 students from 14 educational institutions in metropolitan Lima, 7 from private institutions and 7 public institutions of both sexes. Such analysis indicated that scores of PROLEC-R adapted to be reliable, calculated by the method of internal consistency (Cronbach's alpha) for sub test and total. Also validates evidence of the content (expert opinion), criterion (concurrent validity) and construct (confirmatory factor analysis).

Keywords: Reading, reading processes, reliability, validity 
Leer es una actividad compleja y trascendental para la vida del ser humano, ya que junto al aprendizaje del lenguaje oral, posibilita el dominio e interacción con la cultura y el acceso al conocimiento (González, 2002). Bolaños y Gómez (2009); citando a Meneses (2000) y Vallés, Roig y Navarra (2002), afirman que la adquisición de la lectura es un proceso complejo que se desarrolla por etapas y en el cual se ven involucradas diversas estrategias que implican el desarrollo de habilidades cognitivas que el lector realiza para transitar de la decodificación a la comprensión.

De este modo, para alcanzar la lectura comprensiva, el lector debe transitar por diferentes procesos cognitivos que, considerando el nivel de complejidad, se agrupan en procesos básicos y superiores (Cuetos, 2005; García, 1993; Moreno, Suárez \& Rabazo, 2008). Entre aquellos que forman parte del nivel más elemental de la lectura se encuentran los procesos perceptivos y léxicos. En cambio, los procesos de nivel superior están conformados por los procesos sintácticos y semánticos.

Pese a la importancia de este proceso cognitivo, las evaluaciones realizadas a los estudiantes peruanos sobre el logro de esta habilidad fundamental evidencian un nivel de desempeño inferior al esperado. Ante esto, el Ministerio de Educación (MINEDU) en el año 2005, entre muchas de las alternativas para revertir esta problemática, diseñó un plan de emergencia educativa con énfasis en el fomento del placer por la lectura. No obstante, después de cinco años, las evaluaciones internacionales no evidenciaron avances significativos en la comprensión lectora de nuestros estudiantes, por lo cual cabe preguntarse si es suficiente incentivar el hábito lector o si es necesario indagar sobre los procesos cognitivos que podrían estar ocasionando dichas dificultades.

\section{La Batería de Evaluación de los Procesos Lectores Revisada (PROLEC-R)} es un instrumento de evaluación cuyo objetivo es detectar aquellos procesos cognitivos involucrados en esta habilidad que pueden encontrarse afectados. Por tanto, contar con este instrumento adaptado y baremado sería un aporte importante en el propósito de dotar a los especialistas de aprendizaje y lectura de una herramienta de diagnóstico para la detección temprana y oportuna, y la intervención específica cuando se presentan dificultades. Este es el objetivo que persigue el presente estudio. 


\section{Procesos de la lectura.}

\section{Perceptivos.}

A decir de Domínguez, Cuetos y Vega (2001); Galve (2007) y Moreno et al. (2008), los procesos perceptivos analizan los rasgos de la señal gráfica para hacer su categorización, es decir, ubicarla en el vocabulario léxico del lector. Este análisis visual incluye la discriminación visual, la atención y el almacenamiento en la memoria sensorial breve o icónica, desde la cual la información más relevante pasa a la memoria de corto plazo para su posterior análisis (Galve, 2007; Pino \& Bravo, 2005; Ramos, 2004a).

El reconocimiento de las palabras demanda, además, ciertos movimientos oculares que permiten la adecuada recopilación de la información. Los movimientos sacádicos, que son pequeños saltos de izquierda a derecha que pueden durar entre 25 y 50 milisegundos. Las fijaciones oculares, que son especies de pausas de 200 y 250 milisegundos, en las que el lector fija su mirada en una región del texto para recoger la información. Según Mayer (2002) en las fijaciones se abarca la lectura de seis a ocho letras y el tiempo que los ojos están detenidos dependerá de la familiaridad que se tenga con la palabra. Y las regresiones, que son movimientos que van en dirección contraria a la lectura con la intención de releer una palabra que puede presentar mayor dificultad (palabra nueva, larga o rara).

\section{Léxicos.}

El proceso léxico es el encargado de reconocer la palabra escrita, que operará de diferente manera dependiendo de la familiaridad que se tenga con el vocablo. Existen dos rutas o vías que permiten el reconocimiento de una palabra, la ruta léxica y la ruta fonológica (Cuetos, 2008; Galve, 2007; García, 1993; García, 2006; Moreno et al., 2008; Ramos, 2004a ; Ramos, 2004b). La vía léxica, también llamada directa o visual, permite al lector comparar las características ortográficas de la palabra leída con las representaciones que posee en su memoria léxica, de tal manera que el lector identifica la palabra analizada y activa su significado.

En cambio, cuando se emplea la vía fonológica, indirecta o conocida también como sub-léxica, se convierte cada una de las letras que compone 
la palabra en el sonido que le corresponde, para ello se emplean reglas de conversión grafema-fonema. Subsiguientemente, el lector emplea el ensamblaje fonológico para unir los sonidos formando unidades silábicas articuladas que le permiten acceder al reconocimiento de la palabra en su almacén léxico.

\section{Sintácticos.}

Durante este proceso se analiza la forma o estructura de la oración, es decir, se identifican sus componentes (sujeto, verbo, etc.), se determina qué palabras son de contenido o de función, se analiza el orden de las mismas, se detalla la estructura y las relaciones que se dan entre estos componentes y se lee respetando los signos de puntuación para evitar la ambigüedad del texto (García, 1993). Moreno et al. (2008) explican que durante el proceso sintáctico se analiza el código formal de combinaciones de las unidades lingüísticas, a fin de que el lector haga una representación e interpretación de los hechos o las intenciones comunicativas que propone el autor.

Para alcanzar la comprensión de una oración no basta el análisis sintáctico, ya que este es un primer estadio de análisis superficial que no brinda significación léxica ni proposicional, además opera de forma inconsciente lo que hace imposible la autocorrección. El encargado de complementar el análisis sintáctico es el procesador semántico, éste realiza una compleja interrelación entre la estructura sintáctica y el significado de las palabras, orienta el análisis $\mathrm{y}$, de ser el caso, exige reiterar el primer estadio.

\section{Semánticos.}

Durante el procesamiento de la información de un texto escrito, el lector inicialmente identifica el significado de las palabras, luego de las oraciones y finalmente accederá a la comprensión del texto en su conjunto. La comprensión del discurso escrito equivale a abstraer las proposiciones explícitas e implícitas que presentan las oraciones y relacionarlas con los conocimientos previos del lector, de forma que se logre una nueva construcción de ideas (García, 2006; Meneghetti, Carretti, De Beni, Cornoldi \& Abusamra, 2009; Moreno et al., 2008). 
La primera operación del proceso semántico, la extracción del significado, es superficial porque solo se llevará a la memoria la comprensión explícita del texto. En cambio, la integración del significado de la información implícita es más significativa, en cuanto que la comprensión de un texto, desde este punto, supone la integración de sus elementos individuales y la conexión de esta integración con los saberes previos del lector, alcanzando así un nivel de comprensión más profundo y global que permite la construcción de un modelo mental o situacional coherente del contenido del mismo (Abusamra, Cartoceti, Ferreres, De Beni \& Cornoldi, 2009; Meneghetti et al., 2009).

Teniendo en cuenta una concepción interactiva, todos estos procesos operan mutuamente, puesto que todos están interconectados e influyen uno sobre otro de diferentes formas, de abajo hacia arriba (de procesos de bajo nivel a niveles superiores) y de arriba hacia abajo, dando lugar a la comprensión (Molina, 2008).

De acuerdo a los avances que se han producido sobre la naturaleza del acto lector y la interacción de sus diferentes procesos, el enfoque cognitivo de la lectura considera que su evaluación debe estar mediatizada por la valoración del procesamiento de información que opera sobre los distintos tipos de representaciones lingüísticas. Desde este punto de vista, se trata de averiguar qué sistemas funcionan adecuadamente y cuáles no (Galve, 2007), ya que la intervención temprana de estas deficiencias permitiría incluso disminuir el retraso lector inicial según lo planteado por Vellutino y Scanlon (2002) en Bravo, Villalón \& Orellana (2004).

Ante esto, la Batería de Evaluación de los Procesos Lectores (PROLEC) aplicada en niños y niñas de educación primaria, se ha convertido en un instrumento valioso en la evaluación de las capacidades de lectura, y con mayor importancia en la detección y diagnóstico de los problemas que puede manifestar esta población con respecto a la lectura. El PROLEC es un instrumento que se distingue de muchos otros porque no solo se dirige a evaluar el rendimiento de los estudiantes y establecer así un nivel determinado, sino -y en ello estriba su mayor riqueza- busca indagar en los diferentes procesos que intervienen en la lectura, de esta forma contribuye a la identificación de los aspectos más específicos que podrían estar alterados, para luego plantear acciones específicas que ayuden a superar estas dificultades. 
Es en este sentido y contexto, luego de una primera experiencia y utilización del PROLEC, que ha surgido una versión revisada (PROLEC-R) en el año 2007, a cargo de Fernando Cuetos, Blanca Rodríguez, Elvira Ruano y David Arribas. Pese al difundido uso de esta versión en Lima-Perú, no se contaba aún con la debida adaptación y estandarización que requiere este instrumento, sabiendo de la enorme contribución que ello supondría para el campo de la pedagogía, psicopedagogía y la psicología de nuestro medio.

\section{Objetivos del estudio.}

El objetivo general previsto para el estudio es adaptar y estandarizar el PROLEC-R para alumnos de primaria de Lima Metropolitana. De este propósito general se desprenden objetivos específicos como los siguientes:

1. Adaptar lingüísticamente y pictográficamente el PROLEC-R para alumnos de primaria de Lima Metropolitana.

2. Estimar la confiabilidad mediante la técnica de la consistencia interna del PROLEC-R adaptado.

3. Obtener evidencias de validez de constructo del PROLEC-R adaptado.

4. Elaborar baremos para la interpretación del PROLEC-R adaptado en alumnos de primaria de Lima Metropolitana.

\section{Método}

La investigación corresponde a un estudio psicométrico (Alarcón, 2008; Hernández, Fernández \& Baptista, 2010; Sánchez \& Reyes, 2006). Se analiza las propiedades psicométricas del PROLEC-R adaptado a estudiantes de primaria pertenecientes a instituciones educativas privadas y estatales de Lima Metropolitana.

\section{Participantes.}

En el estudio participaron 504 estudiantes de educación primaria. De este grupo, 252 provienen de instituciones educativas particulares y 252 de estatales, todas ellas de Lima Metropolitana. Del mismo modo, el $50 \%$ de la muestra pertenece al género femenino y el resto al género masculino. Se han considerado un total de 84 estudiantes para cada uno de los seis grados 
escolares, además de hacer una selección equitativa en género (véase tabla 1 y tabla 2).

Tabla 1.

Composición de la muestra según género y tipo de gestión educativa

\begin{tabular}{ccc}
\hline Género & \multicolumn{2}{c}{ Tipo de Gestión } \\
\cline { 2 - 3 } & Particular & Estatal \\
\hline Femenino & 126 & 126 \\
Masculino & 126 & 126 \\
\hline$N=504$
\end{tabular}

$N=504$

Tabla 2.

Distribución de la muestra según grado escolar y género

\begin{tabular}{|c|c|c|c|c|c|}
\hline \multirow[t]{3}{*}{ Grado escolar } & \multicolumn{5}{|c|}{ Género } \\
\hline & \multirow{2}{*}{$\begin{array}{c}\text { Masculino } \\
\mathrm{f}\end{array}$} & \multicolumn{3}{|c|}{ Femenino } & \multirow{2}{*}{ Total } \\
\hline & & $\%$ & $f$ & $\%$ & \\
\hline Primer grado & 42 & 50 & 42 & 50 & 84 \\
\hline Segundo grado & 42 & 50 & 42 & 50 & 84 \\
\hline Tercer grado & 42 & 50 & 42 & 50 & 84 \\
\hline Cuarto grado & 42 & 50 & 42 & 50 & 84 \\
\hline Quintogrado & 42 & 50 & 42 & 50 & 84 \\
\hline Sexto grado & 42 & 50 & 42 & 50 & 84 \\
\hline
\end{tabular}

$\mathrm{N}=252$

El tipo de muestreo utilizado es el probabilístico por conglomerados polietápico, siendo la primera unidad de análisis las Unidades de Gestión Educativa Local (UGEL) de Lima Metropolitana y la segunda unidad de análisis los 14 colegios. Posteriormente, en la tercera unidad de análisis se eligieron las aulas y, finalmente, en la cuarta unidad de análisis se escogieron los sujetos de la muestra. El tamaño de la muestra se determinó con un nivel de confianza de $95 \%$ y un margen de error de $+/-4 \%$. 


\section{Instrumento.}

El instrumento empleado ha sido el PROLEC-R de Cuetos, Rodríguez, Ruano y Arribas (2007). La batería está compuesta por nueve tareas que evalúan los procesos lectores mediante nueve índices principales, 10 secundarios y cinco índices de habilidad normal. La evaluación es individual y se puntúa con uno a la respuesta correcta y cero a la incorrecta. El resultado de las puntuaciones permite diagnosticar la presencia de dificultad leve (D) o severa (DD) en los procesos representados por los índices principales y los de precisión secundarios, para determinar la velocidad lectora (de muy lenta a muy rápida), en los índices de velocidad secundarios, y el nivel lector (bajo, medio o alto) en los sujetos con una habilidad de lectura normal.

\section{Procedimientos.}

En un primer momento se realizaron los cambios lingüísticos y pictográficos necesarios para contextualizar el PROLEC-R en estudiantes de Lima Metropolitana. Estos cambios fueron presentados a un grupo de expertos quienes determinaron la validez de contenido requerida a través del índice $V$ de Aiken. Posteriormente se seleccionó la muestra a través de un procedimiento probabilístico, por conglomerados y polietápico, atendiendo a las variables de sexo, grado y tipo de institución educativa. Para ello se seleccionaron 14 colegios, siete particulares y siete estatales, de las UGEL de Lima Metropolitana. Previa recolección de datos, se efectuaron los contactos respectivos con las entidades correspondientes y se capacitó a un equipo de estudiantes universitarios, en su mayoría de psicología, que se encargarían de la aplicación de la prueba. Posteriormente, a un grupo de docentes se les solicitó hacer una valoración sobre el nivel lector de sus estudiantes, a fin de obtener la validez de criterio. Para finalizar, se calificaron manualmente los protocolos, se elaboró la base de datos en Excel, se vertió la información y se hizo el cálculo correspondiente en el programa estadístico SPSS en su versión 17.

\section{Resultados}

Los datos recolectados, empleando la versión adaptada lingüística y pictóricamente del PROLEC-R en el presente estudio, fueron sometidos a un análisis de tipo estadístico para obtener los principales estadísticos 
descriptivos de tendencia central. Se empleó también la estadística inferencial a través del coeficiente de correlación de Pearson, el coeficiente Alfa de Cronbach y un análisis factorial confirmatorio.

\section{Confiabilidad.}

Para estimar empíricamente la confiabilidad del PROLEC-R adaptado, se utilizó el método de consistencia interna para obtener los valores del coeficiente alfa de Cronbach para cada uno de los ítems y para el total de la prueba (tabla 3). El PROLEC-R adaptado alcanzó puntuaciones satisfactorias, donde el puntaje mayor lo obtuvo el total de la prueba con un alfa de .98. La sub-prueba de lectura de palabras obtuvo el mismo puntaje, seguido del subtest de lectura de pseudopalabras con un .96. El menor valor se obtuvo en la sub-prueba de comprensión oral con.61 (inferior a su par español). En términos generales, estos resultados indican que la prueba es fiable y que, debido a la consistencia interna, tiene sentido la suma de sus reactivos para lograr los subtotales y el total.

Tabla 3.

Cálculo de la confiabilidad

\begin{tabular}{ll}
\hline Sub-pruebas & Alfa \\
\hline Nombre letras & .88 \\
Igual - Diferente & .78 \\
L. Palabras & .98 \\
L. Pseudopalabras & .96 \\
Est. Gramaticales & .82 \\
S. Puntuación & .90 \\
C. Oraciones & .91 \\
C. Textos & .84 \\
C. Oral & .61 \\
\hline Total & .98
\end{tabular}




\section{Validez.}

La versión adaptada del PROLEC-R ha obtenido tres fuentes de evidencias de validez, la de contenido, de criterio y constructo (Brown, 1993, citado por Delgado, Escurra \& Torres, 2006). Para la validez de contenido se presentó la batería, con los cambios lingüísticos y pictográficos necesarios, a un grupo de jueces expertos. Los resultados determinaron el valor de la $V$ de Aiken, según el cual los jueces consideran que la prueba refleja el constructo "procesos lectores", es decir, posee validez de contenido (tabla 4).

Tabla 4.

Validez de contenido por criterio de jueces

\begin{tabular}{|c|c|c|}
\hline Criterio & Modificaciones & V de Aiken \\
\hline \multirow{7}{*}{ TÉRMINOS } & "Enfadado" por "molesto" & 1.00 \\
\hline & "Hucha” por "alcancía” & 1.00 \\
\hline & "Tumbado" por "echado" & 1.00 \\
\hline & "Tarta" por "torta" & 1.00 \\
\hline & "Aplastada contra" por "tirada" & .83 \\
\hline & "Tímidos" por "miedosos" & 1.00 \\
\hline & "Redondel" por "círculo" & 1.00 \\
\hline PROTOCOLO & Tarea Igual-Diferente & 1.00 \\
\hline IMÁGENES & "El soldado es más alto que el indio" & 1.00 \\
\hline
\end{tabular}

Se consideró importante abordar la validez de criterio, pues aportaba evidencia sobre la relación de los datos obtenidos con el test y algún criterio externo, como el desempeño en el área evaluada. Para ello, se correlacionaron las puntuaciones obtenidas en la evaluación de 55 estudiantes con la 
categorización que las docentes y los docentes realizaron sobre los niveles de lectura en los que estos estudiantes se hallaban. Como resultado se aprecia una correlación significativa en los diferentes índices (tabla 5).

Tabla 5.

Correlación de los índices del PROLEC-R adaptado con el criterio de los profesores

\begin{tabular}{cccccc}
\hline \multicolumn{2}{c}{ Índices principales } & \multicolumn{2}{c}{ Índices de precisión } & \multicolumn{2}{c}{ Índices de velocidad } \\
\hline NL & $.356^{* *}$ & NL - P & -.176 & NL - V & $-.403^{* *}$ \\
ID & .189 & ID - P & .219 & ID - V & -.120 \\
LP & $.301^{*}$ & LP - P & .118 & LP - V & $-.317^{*}$ \\
LS & $.335^{*}$ & LS - P & .260 & LS - V & $-.338^{*}$ \\
EG & .050 & - & - & - & - \\
SP & .212 & SP - P & .228 & SP - V & -.193 \\
CO & .123 & & & & \\
CT & $.319^{*}$ & & & & \\
CR & $.311^{*}$ & & & & \\
\hline
\end{tabular}

${ }^{*} p<.05,{ }^{* *} p<.001$

NL: Nombre letras
ID: Igual - diferente
LP: Lectura palabras
LS: Lectura pseudopalabras
EG: Estructuras gramaticales

SP: Signos de puntuación.

CO: Comprensión de oraciones

CT: Comprensión de textos
CR: Comprensión oral

P: Precisión

V: Velocidad

Para obtener la validez de constructo se correlacionaron los índices principales y secundarios, alcanzándose en todos los ítems una correlación significativa (tabla 6). Del mismo modo, la correlación entre los índices principales alcanzó niveles de moderado a alto, mientras la correlación entre los índices principales y los de velocidad es mayor a la alcanzada con los índices de precisión, excepto en algunos procesos semánticos y en la escala de estructuras gramaticales. Asimismo, las correlaciones entre los índices de velocidad, por ser altas o muy altas, indican que el buen desempeño en una escala asegura iguales resultados en las escalas restantes. 


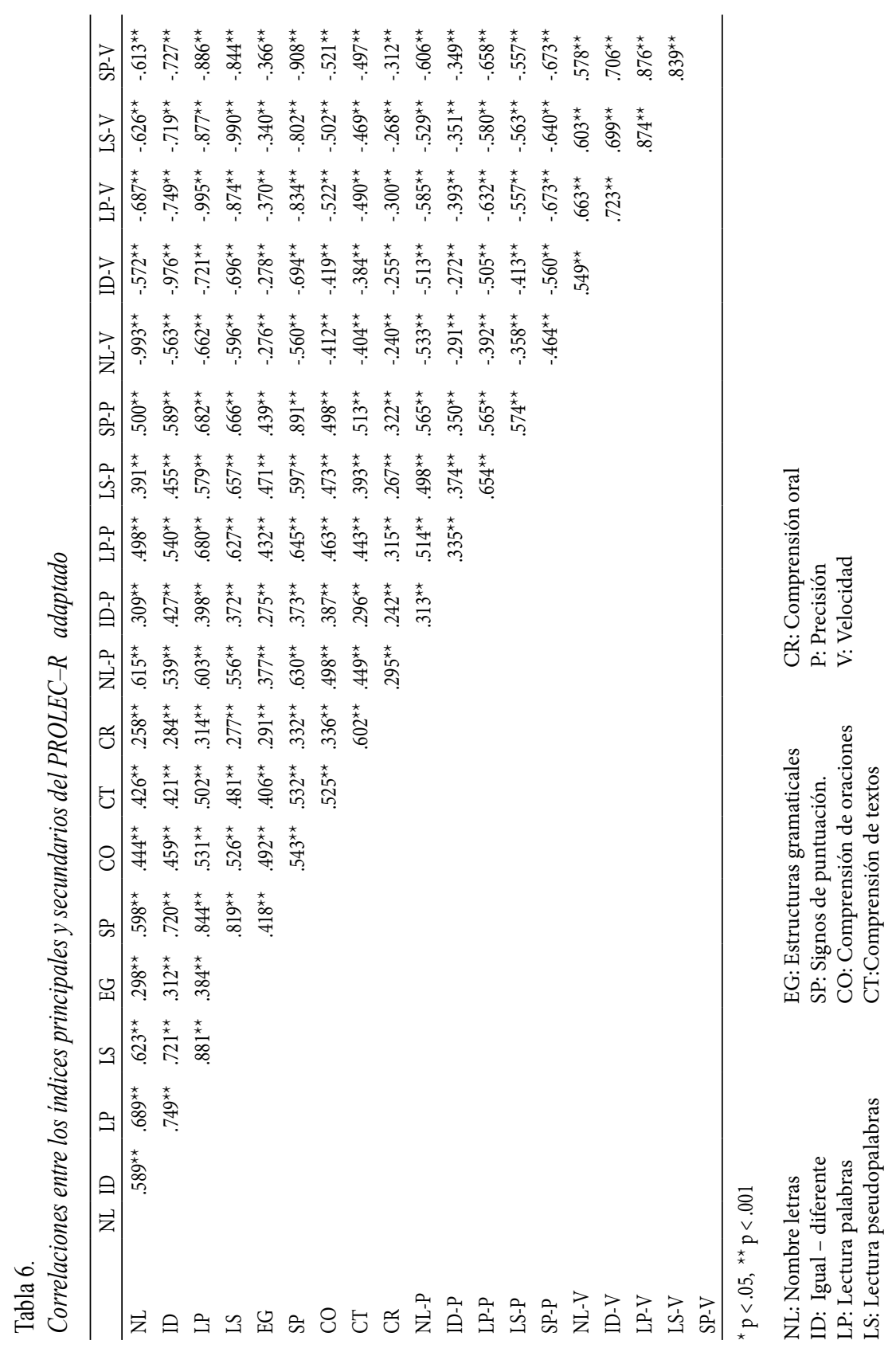


Asimismo, el modelo teórico que subyace a la lectura y a la forma en que se ha estructurado la prueba indica que son cuatro los procesos implicados: identificación de letras, procesos léxicos, procesos sintácticos y procesos semánticos. Esta estructura ha sido puesta a prueba mediante el análisis factorial confirmatorio (véase figura 1), que corrobora la existencia de cuatro variables latentes interrelacionadas entre sí, equivalentes a los cuatro procesos cognitivos del modelo. Estas cuatro dimensiones son las que explicarían el acierto en sus correspondientes tareas. Este modelo dio como resultado un ajuste moderado con las respectivas correlaciones y coeficientes de regresión que aparecen en la figura. En función de estos datos, se puede afirmar que el modelo teórico que subyace al PROLEC- R adaptado se ve reflejado en los datos procedentes de la tipificación, por lo que resulta útil para explicar la conducta lectora de los sujetos.

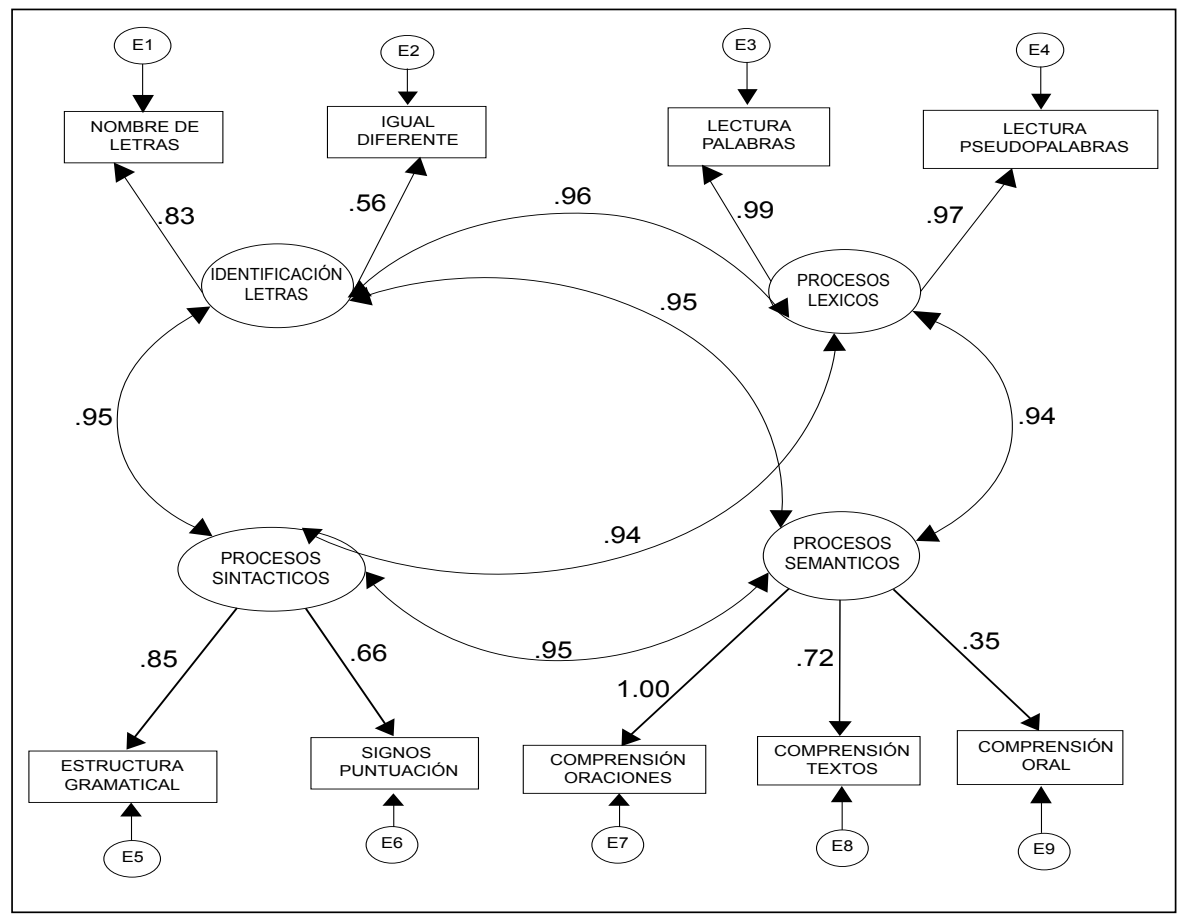

Figura 1. Análisis factorial confirmatorio 


\section{Discusión}

La presente investigación tuvo como principal objetivo la adaptación y estandarización del PROLEC-R en una población de estudiantes de educación primaria de Lima Metropolitana. En ese sentido, pretende ser un aporte a la comunidad de especialistas vinculados a la evaluación y diagnóstico en lectura, aprendizaje y lenguaje, para que puedan contar con un instrumento debidamente adaptado a la realidad de la capital.

El desglose de los resultados, tomando en cuenta la adaptación lingüística y pictográfica, la confiabilidad, la validez y las normas, permite señalar lo siguiente.

La adaptación, revisión y ensayos, en relación al reemplazo de algunos ítems en un sentido lingüístico y pictográfico, fue apoyada por la opinión favorable de jueces expertos. Consideran que en términos de redacción y presentación, estos nuevos ítems representaban la dimensión del constructo procesos lectores. Por tanto, se alcanzó niveles apropiados de validez de contenido, tal como lo indican los valores del coeficiente de Aiken.

Respecto a la validez del PROLEC-R adaptado a Lima, los índices de validez de criterio obtenidos a través de la opinión de los docentes sobre el desempeño lector de los niños y niñas, la intercorrelación de subtest y el análisis factorial confirmatorio, indican que el instrumento tiene índices moderados de validez de constructo, aunque con valores menores a los hallazgos de la versión española.

En lo referente a la confiabilidad estimada con el coeficiente Alfa de Cronbach, los valores indican que el instrumento es confiable para la medición de los procesos lectores. Al respecto, como sucede con la validez, los índices de confiabilidad del PROLEC-R adaptado son similares y, en este caso en particular, incluso ligeramente superiores a los mismos en la versión original.

Las normas de interpretación, por otro lado, se han obtenido de manera semejante a la versión original (adaptación española), conformándose baremos que pretenden ayudar al especialista en aprendizaje y lectura, considerándose que el PROLEC-R es un instrumento de screening respecto al nivel en que se encuentran los procesos lectores de niños y niñas. 
En lo relativo a los datos descriptivos de la muestra, estos indican una evolución de los procesos lectores favorable según el grado escolar. Los resultados son similares también a la muestra española y a los teóricamente esperados, según la naturaleza de la variable de procesos lectores. Este hallazgo, en unión del tiempo de ejecución (a medida que se avanza en grado), indica la influencia no solo de la edad sino también del contexto socioeducativo y familiar, lo cual refuerza la necesidad de una intervención planificada y sistematizada en este aspecto (familia y escuela).

Finalmente, aunque no ha sido objetivo del presente estudio, se observa que el desempeño de los niños de Lima Metropolitana en comparación con sus pares de la versión original, visto a través de los aciertos, medias y desviaciones estándar en cada una de las tareas, es menor. Se obtiene en general puntuaciones más bajas y menores niveles de comprensión, corroborando lo hallado en los diferentes estudios internacionales que se han realizado sobre comprensión lectora en las que se ha incluido al Perú (PISA 2009, SERCE 2008) o estudios nacionales realizados por el MINEDU (UMC, 2009).

\section{Conclusiones}

- El PROLEC-R adaptado a Lima Metropolitana presenta validez de constructo, corroborada a partir de intercorrelación de subtest y de análisis factorial confirmatorio.

- El PROLEC-R adaptado a Lima Metropolitana presenta validez relacionada con el criterio, corroborada mediante la correlación de las puntuaciones obtenidas y la opinión de los docentes sobre el desempeño lector de los niños y niñas evaluados.

- El PROLEC-R adaptado a Lima Metropolitana presenta validez de contenido, evidenciada por la opinión favorable de jueces expertos de las modificaciones lingüísticas y pictográficas realizadas.

- El análisis de confiabilidad, realizado con el método de consistencia interna a través del coeficiente Alfa de Cronbach, confirma la fiabilidad del PROLEC-R adaptado. 
- La versión adaptada del PROLEC-R es una prueba que presenta validez y confiabilidad, que además posee baremos para los estudiantes de educación primaria de Lima Metropolitana, tanto a nivel de índices principales como para los índices secundarios. Por tanto, se concluye en que es un instrumento que permite el diagnóstico con escalas adaptadas a la realidad de los niños y niñas de instituciones educativas de Lima Metropolitana.

\section{Referencias}

Abusamra, V., Cartoceti, R., Ferreres, A., De Bendi, R. \& Cornoldi, C. (2009). La comprensión de textos desde un enfoque multicomponencial. El test "Leer para comprender". Ciencias Psicológicas, 3, 193-200.

Alarcón, R. (2008) Métodos y diseños de Investigación del Comportamiento. Lima: Universidad Ricardo Palma.

Bolaños, R. \& Gómez, L. (2009). Características lectoras de niños con trastorno del aprendizaje de la lectura. Acta Colombiana de Psicología, $12,37-45$.

Bravo, L., Villalón, M. \& Orellana, E. (2004). Procesos cognitivos y el aprendizaje de la lectura inicial: Diferencias cognitivas entre buenos lectores y lectores deficientes. Estudios pedagógicos. 30, 7-19. Recuperado de http://www.scielo.cl/scielo.php?pid=S071807052004000100001\&script $=$ sci_arttext

Cuetos, F. (2005). Psicología de la Lectura. Barcelona: Cisspraxis, S.A.

Cuetos, F., Rodríguez, B., Ruano, E. \& Arribas, D. (2007). PROLEC-R Batería de evaluación de los procesos lectores, revisada. Madrid: TEA Ediciones, S.A.

Cuetos, F. (2008). Psicología de la lectura. Brcelona: Wolters Kluwer S. A.

Delgado, A., Escurra, M. \& Torres, W. (2006). La medición en psicología y educación. Lima: Ediciones HozloS.R.L.

Domínguez, A., Cuetos, F. \& Vega, M. (2001). 100 Palabras polisémicas con sus acepciones. Revista Electrónica de Metodología Aplicada, 6, 63-84. Recuperado de http://www.psico.uniovi.es/rema/v6n2/art2vol6num2.pdf 
Galve, J. (2007). Evaluación e intervención en los procesos de la lectura y escritura. Madrid: Editorial EOS

García, E. (1993). La comprensión de textos: Modelo de procesamiento y estrategias de mejora. Didáctica (lengua y literatura), 5, 87113. Recuperado de http://revistas.ucm.es/edu/11300531/articulos/ DIDA9393110087A.PDF

García, J. (2006). Lectura y conocimiento. Barcelona: Paidós Ibérica.

González, R. (2002). Lectura comprensiva temprana. Lima: Ministerio de Educación.

Hernández, R; Fernández, C y Baptista, P. (2010) Metodología de la Investigación, México DF: McGraw-Hill.

Mayer, R. (2002). Psicología de la educación. Madrid: Pearson Educación S.A.

Meneghetti, C., Carretti, B., De Beni, R., Cornoldi, C. \& Abusamra, A. (2009).

El mejoramiento de la comprensión de texto desde una perspectiva componencial: El caso de la capacidad de individualizar personajes, lugar y tiempo. Ciencias Psicológicas, 3, 185-192.

Ministerio de Educación (2010) Resultados de la Evaluación Censal de Estudiantes ECE 2009. Segundo grado de primaria [presentación Power Point]. Unidad de Medición de la Calidad Educativa (UMC).

Molina, S. (2008). Psicopedagogía de la lengua escrita. Madrid: Editorial EOS.

Moreno, J., Suárez, A. \& Rabazo, Ma. (2008). El Proceso lectoescritor. Estudio de casos. Madrid: Editorial EOS.

OCDE (2010), PISA 2009 Results: What Students Know and Can Do. Student Performance in Reading, Mathematics and Science (Volumen 1), 2010. Recuperado de http://www.pisa.oecd.org/.

Pino, M. \& Bravo, L. (2005). La memoria visual como predictor del aprendizaje de la lectura. Psykhe, 14(1), 47-53. Recuperado de http://www. scielo.cl/scielo.php?pid=S0718-22282005000100004\&script $=$ sci_ arttext\&tlng=es

Ramos, J. (2004a). Ponencia: Una perspectiva cognitiva de las dificultades de lectura y escritura: Procesos, evaluación e intervención. Recuperado de 
http://cprazuaga.juntaextremadura.net/competencias/lengua/primaria/ Ponencia\%20Dificultades\%20lectoescritura.pdf

Ramos, J. (2004b). Enseñar a leer a los alumnos con discapacidad intelectual: Una reflexión sobre la práctica. Revista Ibero Americana, 34, 20-216. Recuperado de http://www.rieoei.org/rie34a07.htm

Sánchez, C.H. \& Reyes, M. C. (2006). Metodología y diseños de la investigación científica. Lima: Visión Universitaria.

Sierra, R. (1988). Técnicas de investigación social. Teoría y ejercicios. Madrid: Paraninfo.

UNESCO - LLECE (2008) Segundo Estudio Regional Comparativo y Explicativo, SERCE [Primer Reporte].

Vallés, E., Roig, J., \& Navarra, J. (2002). Trastornos en el aprendizaje de la lectura. Revista de Logopedia, Foniatría y Audiología, 22, 190-196. 